\section{Atributos y características de los principios orientadores del Modelo de Atención Integral de Salud Familiar y Comunitaria desde la perspectiva de expertos en APS}

\author{
ANGELINA DOIS ${ }^{1, \mathrm{a}}$, PAULINA BRAVO ${ }^{2,3, \mathrm{~b}, \mathrm{~d}}$, GABRIELA SOTO $^{4, \mathrm{c}}$
}

\section{Attributes and features of a community health model from the perspective of practitioners}

Background: The Family and Community Health Model is based on three essential principles: user-centered care, comprehensive care and continuity of care. Aim: To describe the attributes and characteristics of the guiding principles of the Family and Community Health Model (FHM) from the perspective of primary care experts. Material and Methods: This was a qualitative study. An electronic Delphi was conducted with 29 national experts on primary care. Results: The experts agree that user centered care must be based on a psycho-social model integrating the multiple factors that influence health problems. It also must integrate patients' individual features, family and environmental issues. The proposed actions promote shared decision making. To promote integral care, anticipatory guidelines should be expanded and health care of patients with chronic conditions should be improved. Continuity of care should be promoted increasing working hours of medical centers and easing access to integrated electronic medical records, thereby generating efficient links between the different care levels. Conclusions: The results of the study can guide the clinical and administrative management of health teams, allowing the strengthening of primary health care according to the local realities.

(Rev Med Chile 2017; 145: 879-887)

Key words: Continuity of Patient Care; Patient-Centered Care; Primary Health Care.
'Departamento de Salud del Adulto y Senescente, Escuela de Enfermería, Pontificia Universidad Católica de Chile. Santiago, Chile. ${ }^{2}$ Departamento de Salud de la Mujer, Escuela de Enfermería, Pontificia Universidad Católica de Chile. Santiago, Chile.

${ }^{3}$ School of Social Sciences, Cardiff University, UK.

${ }^{4}$ Departamento de Medicina

Familiar, Pontificia Universidad

Católica de Chile. Santiago, Chile.

aEnfermera-matrona, Magíster en

Psicología de la Salud.

bEnfermera.

'Psicóloga, Magíster en Psicología Clínica.

dPhD.

Fuente de apoyo financiero: CONICYT: Fondo Nacional de Investigación en Salud (FONIS), Proyecto FONIS SA14ID0025. FONIS no influyó en el diseño del estudio, en la recolección, análisis o interpretación de los datos ni en la preparación, revisión o aprobación del manuscrito. Las autoras declaran no tener ningún conflicto de interés. Se contó con el apoyo financiero de la DIEE de la Escuela de Enfermería UC para la publicación (costo de revisión) sin que haya influido en la preparación o el contenido del manuscrito.

Recibido el 11 de enero de 2017, aceptado el 26 de julio de 2017.

Correspondencia a:

Angelina Dois

Av. Vicuña Mackenna 4860,

Macul, Santiago, Chile.

adois@uc.cl.
E 1 Modelo de Atención Integral de Salud Familiar y Comunitaria se basa en tres principios irrenunciables: atención centrada en los usuarios (ACU), integralidad de la atención y continuidad del cuidado ${ }^{1,2}$.

La ACU integra aspectos biológicos, emocionales, contextuales y las expectativas de los usuarios ${ }^{3}$, considera la corresponsabilidad del cuidado de la salud entre el equipo profesional y las personas (familias y comunidad), enfatiza los derechos y deberes de los usuarios involucrados y otorga la información necesaria para que las personas puedan participar en las decisiones relativas a su cuidado ${ }^{4}$.

La integralidad de la atención considera el suministro continuo, articulado y de calidad de un rango amplio de prestaciones a lo largo del ciclo vital, para las personas, sus familias y la comunidad en que se insertan ${ }^{2,5}$. Además, incorpora el sistema de creencias asociados a los problemas de salud y a su tratamiento, y el rol que la familia 
juega en el desarrollo de los problemas de salud. Los sistemas de salud que respetan este principio debieran garantizar la atención en los tres niveles decomplejidad, articular las acciones de promoción, prevención, curación y rehabilitación y abordar integralmente a la persona y su familia ${ }^{6,7}$.

La continuidad de la atención se relaciona con el grado en que una serie de eventos de cuidado de la salud son experimentados por las personas como coherentes y conectados entre sí en el tiempo. Implica asumir la responsabilidad del cuidado en distintos escenarios a lo largo de la vida y en el continuo de la red de salud (desde el domicilio a los centros hospitalarios) $)^{2}$. Desde la perspectiva de los proveedores, implica contar con la información y conocimientos suficientes sobre el usuario de modo de poder aplicar sus mejores competencias profesionales y tener la seguridad que la atención será reconocida y continuada por otros proveedores ${ }^{8}$.

Dado que en Chile este ha sido un tema poco explorado, el objetivo de este artículo es describir los atributos y características de los principios orientadores del Modelo de Atención Integral de Salud Familiar y Comunitaria (MAIS) desde la perspectiva de expertos en APS. Los resultados que se presentan forman parte del proyecto Construcción de indicadores de evaluación de los principios que orientan el Modelo de Atención Integral de Salud Familiar y Comunitaria en el ámbito de la Atención Primaria en Salud a través del método Delphi (FONIS SA14ID0025).

\section{Material y Método}

Estudio de diseño cualitativo. Se realizó un Delphi electrónico ${ }^{9}$ con profesionales nacionales expertos en APS.

Para la elección del grupo de expertos del Delphi, se consideraron los criterios de la European Aid Cooperation Office ${ }^{10}$. Los criterios de inclusión incluían a profesionales de la salud que trabajaran en APS al menos los últimos 5 años, jefes de Servicios de Salud, jefes de área de salud de Corporaciones Municipales, directores de Centros de APS y académicos que hubieran desarrollado líneas de trabajo e investigación vinculadas a la APS. El marco muestral inicial estuvo formado por 78 expertos nacionales de reconocida experiencia profesional seleccionados a través de muestreo por conveniencia y en cadena. Una vez definido el universo inicial se determinó el Coeficiente de Competencia de Experto (Kcomp). Este es un método en el que se aplica un cuestionario de autoevaluación respecto del grado de conocimiento sobre el tema en el que se le ha pedido que actúe como experto y las fuentes de donde obtuvo dicho conocimiento. El coeficiente de competencia (Kcomp) se calcula con la fórmula Kcomp $=1 / 2$ $(\mathrm{kc}+\mathrm{ka})$ donde Kc representa el coeficiente de conocimiento o información que tiene el sujeto acerca del problema determinado sobre un total de nueve aspectos referidos al tema y Ka se refiere al coeficiente de argumentación que resulta de sumar los grados de influencia que el sujeto considera que distintas fuentes de argumentación han tenido en el conocimiento acumulado por él respecto de un tema en particular. Se consideró como criterio de selección un valor $\geq 0,8$, es decir, coeficiente de competencia alto ${ }^{10}$. Lo que redujo el grupo de trabajo a 29 expertos. Cada uno de ellos recibió a través de una plataforma virtual, un cuestionario sobre atributos y características de los principios orientadores del MAIS construido usando la evidencia disponible y los resultados del análisis de los grupos focales de la fase inicial del proyecto ${ }^{11}$.

Se realizaron tres rondas de cuestionarios semi estructurados. Al concluir cada ronda, los resultados fueron analizados por los investigadores, utilizando el método de análisis de contenido y devueltos a los participantes. El objetivo del segundo y tercer cuestionario fue consolidar y validar los resultados obtenidos en la consulta previa, solicitar a los expertos que indicaran su grado de acuerdo con la propuesta y que argumentaran sus razones en el caso de no estarlo.

Las tasas de respuesta para cada cuestionario fueron de $72,4 \%, 79,3 \%$ y $86,2 \%$ respectivamente.

Los criterios para finalizar el proceso fueron la convergencia de las estimaciones individuales en un mínimo de $80 \%$ y la estabilidad de las repuestas, que se alcanzó en la tercera ronda de respuestas. Para determinar el nivel de consenso de las respuestas se utilizó el Coeficiente de Concordancia $\left(\mathrm{C}^{3}\right) \geq 75 \%$. El $\mathrm{C}^{3}$ alcanzado varió en un rango entre $83 \%$ y $100 \%$ para el total de los ítems consultados. Para el análisis cuantitativo se usaron medidas de tendencia central y de dispersión de los valores asignados a cada reactivo por cada experto, reordenados en función de los valores medios obtenidos. 
El Consentimiento Informado que resguarda la confidencialidad de la información, la autonomía y la dignidad de los participantes fue aprobado por el Comité Ético-Científico de la Facultad de Medicina de la Pontificia Universidad Católica de Chile (código 47-334) y del Comité de Ética del Servicio Metropolitano Sur Oriente. Todos los participantes firmaron Consentimiento Informado.

\section{Resultados}

La edad promedio de los participantes fue 46 años, en su mayoría médicos, con un tiempo promedio de ejercicio profesional en APS de 19 años (Tabla 1).

A continuación, se detallan los principales resultados obtenidos del análisis, de acuerdo al

Tabla 1. Descripción de los expertos participantes

\begin{tabular}{|c|c|c|c|c|c|c|c|}
\hline & \multirow[t]{2}{*}{ Número respuestas } & \multicolumn{2}{|c|}{ Ronda 1} & \multicolumn{2}{|c|}{ Ronda 2} & \multicolumn{2}{|c|}{ Ronda 3} \\
\hline & & $\mathbf{n}$ & $\%$ & $\mathbf{n}$ & $\%$ & $\mathbf{n}$ & $\%$ \\
\hline \multirow[t]{3}{*}{ Género } & Femenino & 16 & 76,2 & 19 & 82,6 & 20 & 80 \\
\hline & Masculino & 5 & 23,8 & 4 & 17,4 & 5 & 20 \\
\hline & Total & 21 & 100 & 23 & 100 & 25 & 100 \\
\hline \multirow[t]{4}{*}{ Rango Edad (años) } & $31-40$ & 7 & 33,33 & 8 & 36,4 & 11 & 44 \\
\hline & $41-50$ & 7 & 33,33 & 7 & 31,8 & 7 & 28 \\
\hline & $51-60$ & 7 & 33,33 & 7 & 31,8 & 7 & 28 \\
\hline & Total & 21 & 100 & 22 & 100 & 25 & 100 \\
\hline \multirow[t]{11}{*}{ Profesión } & Médico & 1 & 4,76 & 0 & 0,00 & 2 & 8 \\
\hline & Médico Familiar & 11 & 52,38 & 13 & 56,52 & 11 & 44 \\
\hline & Enfermero (a) & 5 & 23,81 & 3 & 13,04 & 5 & 20 \\
\hline & Psicólogo (a) & 2 & 9,52 & 2 & 8,7 & 2 & 8 \\
\hline & Odontólogo (a) & 0 & 0,00 & 1 & 4,34 & 0 & 0 \\
\hline & Asistente Social & 0 & 0,00 & 0 & 0,0 & 0 & 0 \\
\hline & Nutricionista & 1 & 4,76 & 1 & 4,34 & 3 & 12 \\
\hline & Terapeuta ocupacional & 0 & 0,00 & 1 & 4,34 & 0 & 0 \\
\hline & Matrón (a) & 0 & 0,00 & 1 & 4,34 & 0 & 0 \\
\hline & Otro & 1 & 4,76 & 1 & 4,34 & 2 & 8 \\
\hline & Total & 21 & 100 & 23 & 100 & 25 & 100 \\
\hline \multirow{9}{*}{$\begin{array}{l}\text { Área de desempeño } \\
\text { (seleccione todas las que } \\
\text { corresponden) }\end{array}$} & Urbano & 14 & 24,13 & 13 & 24,5 & 15 & 23,4 \\
\hline & Rural & 4 & 6,9 & 2 & 3,8 & 3 & 4,7 \\
\hline & Clínica de adultos & 4 & 6,9 & 4 & 7,5 & 4 & 6,3 \\
\hline & Clínica de niños & 5 & 8,6 & 3 & 5,7 & 8 & 12,5 \\
\hline & Investigación & 2 & 3,5 & 3 & 5,7 & 3 & 4,7 \\
\hline & Gestión & 16 & 27,6 & 15 & 28,3 & 15 & 23,4 \\
\hline & Docencia & 12 & 20,7 & 12 & 22,6 & 16 & 25 \\
\hline & Otra área & 1 & 1,7 & 1 & 1,9 & 0 & 0 \\
\hline & Total & 58 & & 53 & & 64 & \\
\hline \multirow{5}{*}{$\begin{array}{l}\text { Tiempo de experiencia en APS } \\
\text { (años) }\end{array}$} & $5-10$ & 5 & 23,81 & 6 & 27,3 & 8 & 32 \\
\hline & $11-20$ & 8 & 38,10 & 9 & 40,9 & 7 & 28 \\
\hline & $21-30$ & 7 & 33,33 & 5 & 22,7 & 4 & 16 \\
\hline & $31-40$ & 1 & 4,76 & 2 & 9,1 & 6 & 24 \\
\hline & Total & 21 & 100 & 22 & 100 & 25 & 100 \\
\hline \multirow{7}{*}{$\begin{array}{l}\text { Área geográfica en que ha } \\
\text { desarrollado su trabajo en APS } \\
\text { (seleccione todas las que } \\
\text { corresponden) }\end{array}$} & $\mathrm{RM}$ & 15 & 51,7 & 14 & 63,7 & 18 & 72 \\
\hline & IV región & 2 & 6,9 & 2 & 9,1 & 1 & 4 \\
\hline & VI región & 1 & 3,5 & 1 & 4,5 & 0 & 0 \\
\hline & VIII región & 3 & 10,3 & 2 & 9,1 & 4 & 16 \\
\hline & IX región & 6 & 20,7 & 2 & 9,1 & 2 & 8 \\
\hline & X región & 2 & 6,9 & 1 & 4,5 & 0 & 0 \\
\hline & Total & 29 & 100 & 22 & 100 & 25 & 100 \\
\hline
\end{tabular}


principio orientador, los atributos de cada uno de ellos, y acciones relacionadas desde la perspectiva de los expertos en APS.

\section{Atención centrada en el usuario}

Los expertos concuerdan que debe organizarse desde un enfoque terapéutico biopsicosocial que integre la multi-causalidad de los problemas de salud o del motivo de consulta del usuario, las características individuales y del entorno en el que está inserto y el rol dela familia en la salud de sus integrantes (Tabla 2). Entre las acciones propuestas se consideran los aspectos relacionales que permitan establecer una alianza con el usuario y su familia (cuando corresponda). Esto se vería reflejado al considerar durante la atención de salud las preferencias y valores de las personas y al otorgarles la información necesaria para que participen activamente en la toma de decisiones relativas a su cuidado (Tabla 3 ).

Tabla 2. Atributos de la atención centrada en la persona

\begin{tabular}{|lcc|c|}
\hline Atributo & $\begin{array}{c}\text { Muy de } \\
\text { acuerdo }\end{array}$ & $\begin{array}{c}\text { Percentaje (\%) } \\
\text { acuerdo }\end{array}$ & $\begin{array}{c}\text { Ni de acuerdo En desacuerdo } \\
\text { ni en } \\
\text { desacuerdo }\end{array}$ \\
$\begin{array}{c}\text { o muy en } \\
\text { desacuerdo }\end{array}$ \\
$\begin{array}{l}\text { Los profesionales de la salud consideran en el enfoque terapéuti- } \\
\begin{array}{l}\text { co la multicausalidad de los problemas de salud o del motivo de } \\
\text { consulta del usuario }\end{array}\end{array}$ & 95,3 & 4,7 & 0 \\
$\begin{array}{l}\text { Los profesionales de la salud consideran a la familia como un } \\
\text { factor que pueda afectar positiva o negativamente la salud de sus } \\
\text { miembros }\end{array}$ & 100 & 0 & 0 \\
$\begin{array}{l}\text { Los profesionales de la salud emplean en la atención del usuario } \\
\text { el enfoque biopsicosocial }\end{array}$ & 100 & 0 & 0 \\
$\begin{array}{l}\text { Los profesionales de la salud emplean en el análisis de la experien- } \\
\text { cia del usuario una visión integral considerando sus características } \\
\text { individuales y el entorno en el que está inserto }\end{array}$ & 90,5 & 9,5 & 0 \\
\hline
\end{tabular}

Tabla 3. Acciones a considerar en una consulta de salud centrada en el usuario

\begin{tabular}{|c|c|c|c|c|}
\hline \multirow[t]{2}{*}{ Acciones } & \multicolumn{4}{|c|}{ Porcentaje (\%) } \\
\hline & $\begin{array}{l}\text { Muy de } \\
\text { acuerdo }\end{array}$ & $\begin{array}{l}\text { De } \\
\text { acuerdo }\end{array}$ & $\begin{array}{c}\text { Ni de acuerdo } \\
\text { ni en } \\
\text { desacuerdo }\end{array}$ & $\begin{array}{c}\text { En desacuerdo } \\
\text { o muy en } \\
\text { desacuerdo }\end{array}$ \\
\hline Saluda al usuario al inicio del encuentro & 100 & 0 & 0 & 0 \\
\hline Se dirige al usuario por su nombre & 81 & 19 & 0 & 0 \\
\hline Mira al usuario a los ojos mientras le habla & 90,5 & 9,5 & 0 & 0 \\
\hline $\begin{array}{l}\text { Establece relaciones terapéuticas que demuestran coherencia, } \\
\text { empatía y aceptación del usuario }\end{array}$ & 100 & 0 & 0 & 0 \\
\hline Escucha activamente lo que plantea el usuario & 100 & 0 & 0 & 0 \\
\hline $\begin{array}{l}\text { Considera en la atención de salud las preferencias y valores de los } \\
\text { usuarios }\end{array}$ & 85,7 & 14,3 & 0 & 0 \\
\hline $\begin{array}{l}\text { El profesional establece una alianza con el usuario y su familia (si } \\
\text { corresponde) }\end{array}$ & 85,7 & 14,3 & 0 & 0 \\
\hline $\begin{array}{l}\text { El profesional evalúa las necesidades de información que el usuario } \\
\text { pueda tener para tomar una decisión relativa a su salud }\end{array}$ & 95.2 & 4,8 & 0 & 0 \\
\hline $\begin{array}{l}\text { El profesional entrega información al usuario que sea comprensible } \\
\text { y pertinente al motivo de consulta }\end{array}$ & 100 & 0 & 0 & 0 \\
\hline $\begin{array}{l}\text { El profesional promueve la participación activa de los usuarios en } \\
\text { la toma de decisiones que involucran su cuidado }\end{array}$ & 100 & 0 & 0 & 0 \\
\hline
\end{tabular}




\section{Integralidad}

Los expertos concuerdan que sus atributos característicos son la promoción y prevención en salud, y la participación. En relación a lo promocional y preventivo proponen ampliar la oferta a Guías Anticipatorias (Tabla 4), pero reconocen que su efectividad considera acciones interactivas entre el profesional y el usuario, por lo que no puede limitarse a la mera entrega del material impreso. Por ello, proponen incluir capacitación en relación a los contenidos de la guía y la forma de utilizarla durante el encuentro clínico de forma que el profesional pueda rescatar la individualidad del usuario, sus conocimientos y su contexto biográfico. En relación a la atención de personas con problemas crónicos, existe consenso que es necesario aumentar el tiempo destinado al control de salud (Tabla 5), 90,4\% considera necesario que participen trabajadores sociales y psicólogos como miembros permanentes del equipo a cargo y, por último, plantean la necesidad de extender la participación familiar a controles, acciones de monitoreo en hogar e instancias educativas.

Tabla 4. Propuesta de Guías anticipatorias

\begin{tabular}{|c|c|}
\hline \multicolumn{2}{|c|}{ Propuesta de Guías Promocionales y Preventivas para la Atención Primaria } \\
\hline Grupo & Tema \\
\hline Embarazadas & 1. Guía de la gestación Chile Crece Contigo \\
\hline $\begin{array}{l}\text { Adultos responsables } \\
\text { de niños }<1 \text { año }\end{array}$ & $\begin{array}{l}\text { 1. Fomento alimentación saludable: lactancia materna } \\
\text { 2. Cuidados del crecimiento y desarrollo } \\
\text { 3. Estimulación del desarrollo psicomotor }\end{array}$ \\
\hline $\begin{array}{l}\text { Adultos responsables } \\
\text { de niños entre } \\
1 \text { y } 5 \text { años }\end{array}$ & $\begin{array}{l}\text { 1. Alimentación saludable } \\
\text { 2. Prevención de accidentes } \\
\text { 3. Estilos de vida saludable } \\
\text { 4. Pautas de crianza respetuosa }\end{array}$ \\
\hline $\begin{array}{l}\text { Niños } \\
(6-10 \text { años) }\end{array}$ & $\begin{array}{l}\text { 1. Fomento de actividad física } \\
\text { 2. Fomento Alimentación saludable } \\
\text { 3. Prevención de accidentes }\end{array}$ \\
\hline $\begin{array}{l}\text { Adolescentes } \\
\text { (11-19 años) }\end{array}$ & $\begin{array}{l}\text { 1. Fomento de actividad física } \\
\text { 2. Fomento Alimentación saludable } \\
\text { 3. Salud sexual y reproductiva: prevención del embarazo adolescente, prevención de VIH y otras ITS } \\
\text { y prevención de cáncer cérvico-uterino y VPH } \\
\text { 4. Consumo de sustancias (tabaco, alcohol y otras drogas) } \\
\text { 5. Prevención de accidentes }\end{array}$ \\
\hline $\begin{array}{l}\text { Adultos } \\
\text { (20-64 años) }\end{array}$ & $\begin{array}{l}\text { 1. Fomento de actividad física } \\
\text { 2. Fomento Alimentación saludable } \\
\text { 3. Consumo responsable de alcohol, tabaco y drogas } \\
\text { 4. Salud sexual y reproductiva: prevención de VIH y otras ITS, planificación familiar, climaterio- } \\
\text { menopausia }\end{array}$ \\
\hline $\begin{array}{l}\text { Adulto mayor } \\
\text { (mayor de } 65 \text { años) }\end{array}$ & $\begin{array}{l}\text { 1. Cambios fisiológicos asociados al envejecimiento } \\
\text { 2. Fomento de actividad física } \\
\text { 3. Fomento alimentación saludable } \\
\text { 4. Fomento de la participación social } \\
\text { 5. Salud sexual y reproductiva: sexualidad en las personas mayores } \\
\text { 6. Prevención de caídas } \\
\text { 7. Ejercicio de memoria }\end{array}$ \\
\hline Ciclo vital familiar & $\begin{array}{l}\text { 1. Etapas del ciclo vital familiar: características, tareas y potenciales conflictos de cada una de las etapas } \\
\text { 2. Cuidado del cuidador de familiares (adultos y niños) con dependencia }\end{array}$ \\
\hline
\end{tabular}




\section{Continuidad}

Los expertos identifican un listado de atributos $y$ acciones que deben estar presentes en la organización de la atención de salud, para dar cuenta de este principio como organizador del MAIS (Tabla 6). Entre las acciones, proponen ajustar las prestaciones ofrecidas organizándolas de acuerdo a grupos de riesgo y a horarios de atención diferidos, lo que es especialmente importante en personas que trabajan fuera de su domicilio o que están a cargo del cuidado de otros y que no pueden concurrir al centro de salud en los horarios habituales de funcionamiento (Tabla 7). Sugieren un plan de instalación progresiva de las prestaciones contempladas en las metas sanitarias durante el horario de extensión de la atención en el Centro de Salud, asegurando el acceso a toda la población sin que esto requiera inversión en infraestructura, sino sólo redistribución de su uso. Esto traería como beneficio secundario la disminución de las consultas en SAPU y Urgencia que correspondan a atenciones no resueltas en el Centro de Salud.

Otro ámbito de acción relacionado con este principio es el acceso a información relevante para la atención de usuarios a través de registros electrónicos, que permita la vinculación de los distintos niveles de atención y con ello la coordinación de las prestaciones (Tabla 8).

Tabla 5. Rendimiento controles de salud de personas con enfermedades crónicas

\begin{tabular}{|lccccc|}
\hline & Muy de acuerdo & De acuerdo & $\begin{array}{c}\text { Porcentaje (\%) } \\
\text { Ni de acuerdo ni } \\
\text { en desacuerdo }\end{array}$ & En desacuerdo & $\begin{array}{c}\text { Totalmente en } \\
\text { desacuerdo }\end{array}$ \\
\hline 4 controles/h & 0 & 9,5 & 9,5 & 19,1 & 61,9 \\
\hline 3 controles/h & 23,8 & 42,9 & 4,7 & 14,3 & 14,3 \\
\hline 2 controles/h & 52,4 & 38,2 & 4,7 & 4,7 & 0 \\
\hline
\end{tabular}

Tabla 6. Atributos y acciones de la continuidad del cuidado

\begin{tabular}{|c|c|c|c|c|}
\hline \multirow[t]{2}{*}{ Atributos-acciones } & \multicolumn{4}{|c|}{ Porcentaje (\%) } \\
\hline & $\begin{array}{l}\text { Muy de } \\
\text { acuerdo }\end{array}$ & $\begin{array}{l}\text { De } \\
\text { acuerdo }\end{array}$ & $\begin{array}{l}\text { Ni de acuerdo } \\
\text { ni en } \\
\text { desacuerdo }\end{array}$ & $\begin{array}{l}\text { En desacuerdo } \\
\text { o muy en } \\
\text { desacuerdo }\end{array}$ \\
\hline $\begin{array}{l}\text { El usuario conoce a los profesionales que conforman su equipo de } \\
\text { salud de cabecera }\end{array}$ & 81 & 14,3 & 4,7 & 0 \\
\hline $\begin{array}{l}\text { Cada equipo de salud de cabecera conoce a los usuarios y familias } \\
\text { que corresponden a su sector }\end{array}$ & 71,4 & 28,6 & 0 & 0 \\
\hline $\begin{array}{l}\text { Cada equipo de salud de cabecera conoce cuales son los territorios } \\
\text { los que pertenecen los usuarios y familias que corresponden a su } \\
\text { sector }\end{array}$ & 76,2 & 19,1 & 4,7 & 0 \\
\hline $\begin{array}{l}\text { Un usuario que presenta una condición crónica de salud puede } \\
\text { acceder a atenderse con el médico que mejor lo conoce }\end{array}$ & 81 & 9,5 & 9,5 & 0 \\
\hline $\begin{array}{l}\text { Un usuario que presenta una condición crónica de salud puede } \\
\text { acceder a atenderse con los profesionales del equipo de salud que } \\
\text { mejor lo conocen }\end{array}$ & 81 & 14,3 & 4,7 & 0 \\
\hline $\begin{array}{l}\text { Un usuario que presenta una condición aguda de morbilidad } \\
\text { puede acceder a atenderse con el médico que mejor lo conoce }\end{array}$ & 28,6 & 57,1 & 14,3 & 0 \\
\hline $\begin{array}{l}\text { Un usuario que presenta una condición aguda de morbilidad pue- } \\
\text { de acceder a atenderse con los profesionales del equipo de salud } \\
\text { que mejor lo conocen }\end{array}$ & 28,6 & 57,1 & 14,3 & 0 \\
\hline
\end{tabular}


Tabla 7. Oferta de prestaciones según horario y grupo

\begin{tabular}{|c|c|c|c|c|}
\hline \multirow[t]{2}{*}{ Acciones } & \multicolumn{4}{|c|}{ Porcentaje (\%) } \\
\hline & $\begin{array}{l}\text { Muy de } \\
\text { acuerdo }\end{array}$ & $\begin{array}{l}\text { De } \\
\text { acuerdo }\end{array}$ & $\begin{array}{l}\text { Ni de acuerdo } \\
\text { ni en } \\
\text { desacuerdo }\end{array}$ & $\begin{array}{l}\text { En desacuerdo } \\
\text { o muy en } \\
\text { desacuerdo }\end{array}$ \\
\hline $\begin{array}{l}\text { Los CESFAM deben ofrecer atenciones de salud en horarios amplios } \\
\text { que permitan dar mayores facilidades de acceso a los usuarios y } \\
\text { priorizar a los grupos de mayor riesgo }\end{array}$ & 90 & 5 & 5 & 0 \\
\hline $\begin{array}{l}\text { LOS CESFAM deben diferir horarios de colación y de entrada y } \\
\text { salida de los profesionales de la salud }\end{array}$ & 75 & 10 & 10 & 5 \\
\hline $\begin{array}{l}\text { Los CESFAM deben contar con cupos reservados de atención para } \\
\text { usuarios de grupos de riesgo }\end{array}$ & 80 & 10 & 5 & 5 \\
\hline
\end{tabular}

Tabla 8. Acceso a información relevante para la atención de usuarios

\begin{tabular}{|c|c|c|c|c|}
\hline \multirow[t]{2}{*}{ Acciones } & \multicolumn{4}{|c|}{ Porcentaje (\%) } \\
\hline & $\begin{array}{l}\text { Muy de } \\
\text { acuerdo }\end{array}$ & $\begin{array}{c}\text { De } \\
\text { acuerdo }\end{array}$ & $\begin{array}{l}\text { Ni de acuerdo } \\
\text { ni en } \\
\text { desacuerdo }\end{array}$ & $\begin{array}{l}\text { En desacuerdo } \\
\text { o muy en } \\
\text { desacuerdo }\end{array}$ \\
\hline $\begin{array}{l}\text { La ficha clínica del usuario debe estar completa y disponible para } \\
\text { todos los profesionales de la salud del equipo involucrado en la } \\
\text { atención }\end{array}$ & 85,71 & 14,29 & 0 & 0 \\
\hline $\begin{array}{l}\text { La ficha clínica del usuario debe estar disponible en cada una de } \\
\text { las atenciones de salud del usuario (programadas o espontáneas) }\end{array}$ & 95,24 & 4,76 & 0 & 0 \\
\hline $\begin{array}{l}\text { El usuario debe tener acceso fácil a la información que requiere } \\
\text { para facilitar su "navegación/tránsito" dentro del sistema de salud }\end{array}$ & 95,2 & 0 & 0 & 4,76 \\
\hline $\begin{array}{l}\text { El profesional que deriva a un usuario a otro nivel de atención } \\
\text { debiera realizar un seguimiento del caso }\end{array}$ & 80 & 10 & 10 & 0 \\
\hline
\end{tabular}

\section{Discusión}

Este artículo describe los atributos y características de los principios orientadores del MAIS desde la perspectiva de expertos en APS y las acciones asociadas a cada uno de ellos.

Los expertos participantes describen como elemento fundamental de la ACU, el trato que los profesionales otorgan a las personas durante la atención de salud y describen acciones similares a las reportadas en otros estudios ${ }^{4,12,13}$. Un ejemplo de ello es la integración de un enfoque biopsicosocial en la atención clínica que considere globalmente la situación del usuario, más allá de la mera patología, lo que permitiría establecer una alianza terapéutica para construir un plan de trabajo conjunto que incorpore la forma particular en que la persona vive su condición de salud ${ }^{3,14}$. Esta perspectiva integradora y centrada en el usuario tiene un potencial organizador de la atención en salud y permite al usuario experimentar una atención en salud transparente, individualizada ${ }^{15}$ y congruente con sus necesidades ${ }^{16}$.

Los expertos describen como atributo de la integralidad las acciones de promoción y prevención en salud y la participación de los usuarios. Entre las acciones propuestas describen la educación en salud a través de guías anticipatorias y el trabajo con personas portadoras de condiciones crónicas de salud. Si bien existe suficiente evidencia acerca de la efectividad de estas intervenciones ${ }^{17}$, una de las dificultades que enfrentan los equipos de salud 
en su implementación es lograr la adherencia de los usuarios a distintas instancias educativas. Esta situación puede asociarse al menos a dos situaciones, una de ellas vinculada al acceso, ya que habitualmente los talleres consideran varias sesiones y coinciden con actividades laborales y/o de otra índole, tanto del usuario como de los familiares. La otra, asociada al foco de la intervención ya que muchas veces las instancias educativas se encuentran protocolizadas de forma de abordar temas que desde la perspectiva de los expertos son obligatorios, pero que no necesariamente se constituyen en temas generativos para los usuarios. Por lo tanto, es relevante considerar el diagnóstico previo de las necesidades de la población objetivo y el uso de metodologías participativas atractivas ${ }^{18,19}$ y que aborden las necesidades sentidas por la población ${ }^{7}$. Así el balance entre los servicios disponibles y los proporcionados se ajustaría a las necesidades específicas de la población por sobre las recomendaciones generales que se hacen para diferentes grupos poblacionales ${ }^{6}$. Las acciones vinculadas a este principio de integralidad interpelan a los profesionales de la APS a incorporar innovaciones en las prestaciones otorgadas y a sobrellevar los desafíos de su implementación tanto en el nivel primario como en sus interacciones con otros componentes del sistema y la sociedad ${ }^{20}$.

Por último, respecto de la continuidad de la atención se plantea la necesidad de ofrecer toda la canasta de prestaciones durante la modalidad extendida de atención. En ese sentido, la evaluación económica y de impacto es requisito indispensable al implementar intervenciones innovadoras, de manera de estimar las necesidades de recursos humanos y económicos para cubrir el personal profesional y no profesional requerido y la evaluación del per cápita asignado.

Otro punto de consenso en relación a la continuidad se refiere a la necesidad de contar con un sistema integrado de información de los usuarios que permita la comunicación entre los distintos niveles de la red y los profesionales responsables de las prestaciones al usuario, condición que fue planteada en 1994 por Barbara Starfield ${ }^{21}$. Este es un elemento clave en el enlace de la atención de salud, que influye directamente en la percepción del usuario sobre la integración o coordinación de los servicios, situación ya reportada por otros autores ${ }^{22,23}$ y que, a juicio de los expertos participantes, se vería favorecida si entre otras medidas, se contara con una ficha clínica única por macrozona y con sistemas eficientes y expeditos de referencia y contrarreferencia.

Para la APS es de suma importancia contar con sistemas de información que permitan hacer seguimiento respecto de lo que ocurre con la población a cargo ${ }^{8,24}$ para asegurar el acceso a una atención de salud oportuna y continua que se ha demostrado impacta directamente en la disminución del gasto en salud y la mejora de la atención preventiva ${ }^{25,26}$.

En síntesis, los resultados del estudio pueden servir para orientar la gestión clínica y administrativa y la praxis habitual en la atención de los equipos de salud en prácticas que permitan el fortalecimiento de la instalación del MAIS en APS de acuerdo a sus principios orientadores y a las distintas realidades locales, los que se vinculan a mejores índices de satisfacción usuaria y de resultados clínicos a mediano y largo plazo como también en la organización y coordinación de los servicios que proveen los prestadores de salud, lo que impacta en la calidad de la atención y la disminución de los costos globales de atención ${ }^{4,15,27}$.

Limitaciones: los resultados obtenidos son el reflejo de la experiencia de un conjunto de profesionales expertos en APS, en su mayoría médicos que se desempeñan en la Región Metropolitana, y si bien comparten elementos con otros estudios en esta línea, los hallazgos no necesariamente son trasferibles a otras realidades.

\section{Referencias}

1. Organización Panamericana de la Salud. Hacia un modelo de atención integral a la salud basado en la Atención Primaria de Salud. Washington DC: Organización Panamericana de la Salud, 2012.

2. Ministerio de Salud. Orientaciones para la implementación del Modelo de Atención Integral de Salud familiar y comunitaria. Chile: Ministerio de Salud, 2013.

3. Coppolillo F. Cuidados centrados en la persona. Bases epistemológicas para la atención primaria y la medicina familiar. Rev arch med familiar gen 2013; 10 (1): 45-50.

4. Robinson J, Callister L, Berry J, Dearing KA. Patient-centered care and adherence: Definitions and applications to improve outcomes. J Am Assoc Nurse Pract 2008; 20: 600-7.

5. Acosta N, Vega R. El caso de la implementación de la estrategia de Atención Primaria Integral en Salud (APIS). Rev. Gerenc. Polit. Salud, Bogotá (Colombia). 
[Internet]. 2008 [citado el 15 de marzo de 2015]; 7 (14), 125-44.Disponible en: http://www.scielo.org.co/pdf/ rgps/v7n14/v7n14a08.pdf

6. Aguilera C. O desafio da integralidade segundo as perspectivas da vigilância da saúde e da saúde da família. Ciênc. Saúde coletiva [Internet]. 2003 [citado el 15 de marzo de 2015]; 8 (2): 569-84. Disponible en http:// www.scielosp.org/scielo.php?script=sci_arttext\&pi$\mathrm{d}=$ S1413-81232003000200018\&lng=en.

7. Rosas A, Zárate V, Cuba MS. Atributos de la Atención Primaria de Salud (A.P.S): Una visión desde la Medicina Familiar. Acta méd peruana [Internet]. 2013 [citado el 6 de marzo de 2015]; 30 (1). Disponible en http:// www.scielo.org.pe/scielo.php?script=sci_arttext\&pi$\mathrm{d}=$ S1728-59172013000100008

8. Slater L. Person-centredness: A concept analysis. Contemp Nurse [Intenet]. 2006 [citado 10 may 2015]; 23: 135-44. Disponible en: http://www.tandfonline.com/ doi/full/10.5555/conu.2006.23.1.135

9. Varela M, Díaz L, García R. Descripción y usos del método Delphi en investigaciones del área de la salud. Inv Ed Med. [Internet]. 2012 [citado el 26 de enero de 2016]; 1 (2): 90-5. Disponible en: http://riem.facmed. unam.mx/sites/all/archivos/V1Num02/07_MI_DESCRIPCION_Y_USOS.PDF

10. García V, Aquino S, Guzmán A, Medina A. El uso del método Delphi como estrategia para la valoración de indicadores de calidad en programas educativos a distancia. Revista CAES [Internet]. 2012 [citado el 6 de abril de 2015]; 3 (1): 200-22. Disponible en: https:// dialnet.unirioja.es/servlet/articulo?codigo $=3945837$

11. Dois A, Contreras A, Bravo P, Mora I, Soto G, Solís C. Principios orientadores del Modelo Integral de Salud Familiar y Comunitario desde la perspectiva de los usuarios. Rev Med Chile [Internet]. 2016 [citado el 26 de diciembre de 2016]; 144 (5): 585-92. Disponible en: http://www.scielo.cl/scielo.php?script=sci_arttext\&pi$\mathrm{d}=$ S0034-98872016000500005\&lng=es.

12. Elo S, Kyngäs $H$. The qualitative content analysis. JAN 2007; 62 (1): 107-15.

13. Beach M, Saha S, Cooper L. The role and relationship of cultural competence and patient-centeredness in health care quality. Commonwealth Fund 2006; (960): 1-22.

14. De la Uz, ME. La Relación médico-paciente y su panorama actual. Bioética [Internet]. 2010 [citado el 6 de diciembre de 2015]: 21-5. Disponible en http://cbioetica. org/revista/101/101-2125.pdf

15. Rathert C, Wyrwich M, Boren S. Patient-Centered Care and Outcomes: A Systematic Review of the literature. Med Care Res 2013; 70: 351-79.

16. Montenegro H, Suárez J. Redes Integradas de Servicios de Salud: conceptos, opciones de política y hoja de ruta para su implementación en las Américas. Washington, D.C.: Organización Panamericana de la Salud, 2010.

17. Prior M, Guerin M, Grimmer-Somers K. The effectiveness of clinical guideline implementation strategies a synthesis of systematic review findings. J Eval Clin Pract. [Internet]. 2008 [citado el 12 de mayo de 2015]; 14: 888-97. Disponible en: http://onlinelibrary.wiley.com. ezproxy.puc.cl/doi/10.1111/j.1365-2753.2008.01014.x/ abstract;jsessionid=3C5418664E2C84B19A7B2E21ED8C3657.f02t02

18. Vella J. Aprender a Hablar de tareas. Estrategias creativas para la enseñanza de adultos. San Francisco: Jossey Bass Publishers; 2000.

19. Montero J. Educación participativa de adultos. El modelo dialogante de Jane Vella. Experiencia en Chile. Ars Médica [Internet]. 2007 [citado el 26 de diciembre de 2016]; 36 (2). Disponible en: http://www.arsmedica.cl/ index.php/MED/article/view/151/91

20. Pinheiro R, Alcindo F, Gomes da A. Integrality in the population's health care programs. Ciênc. saúde coletiva [Internet]. 2007 [citado el 15 de marzo de 2015]; 12 (2): 343-49. Disponible en http://www.scielo.br/scielo. php?pid=S1413-81232007000200010\&script=sci_arttext.

21. Starfield B. Is primary care essential? Lancet 1994; 344: 1129-33.

22. Salisbury C, Sampson F, Ridd M, Montgomery A. How should continuity of care in primary health care be assessed? Br J Gen Pract 2009; 59: 276-28.

23. Morgan S, Yoder LH. A concept analysis of person-centered care. J Holist Nurs 2012; 30 (1): 6-15.

24. Haggerty J, Reid R, Freeman G, Starfield B, Adair C, McKendry R. Continuity of care: a multidisciplinary review. BMJ 2003; 32: 1219-21.

25. Shin D, Cho J, Yang HK, Park JH, Lee H, Kim H, et al. Impact of continuity of care in mortality and health care cost: a nationwide cohort study in Korea. Ann Fam Med 2014; 12: 534-41.

26. Letelier MJ, Aller MB, Henao D, Sánchez-Pérez I, Vargas I. et al. Diseño y validación de un cuestionario para medir la continuidad asistencial entre niveles desde la perspectiva del usuario: CCAENA. Gac Sanit. [Internet]. 2010; [citado el 6 de noviembre de 2015]; 24 (4): 339-46. Disponible en: http://www.gacetasanitaria.org/ es/diseno-validacion-un-cuestionario-medir/articulo/ S0213911110001408/

27. Kitson A, Marshall A, Bassett K, Zeitz K. What are the core elements of patient-centred care? A narrative review and synthesis of the literature from health policy, medicine and nursing. J Adv Nurs 2013; 69 (1): 4-15. 